Петков Александр Александрович

старший научный сотрудник, доктор технических наук, профессор кафедры

«Инженерная электрофизика»

Национальный технический университет «Харьковский политехнический институт», г. Харьков,

Украина

apetkov@yandex.ru

\title{
САМООБУЧАЕМОСТЬ КОМПЬЮТЕРНЫХ ПРОГРАММ КОНТРОЛЯ ВЫПОЛНЕНИЯ ТЕСТОВЫХ ЗАДАНИЙ РАСЧЕТНОГО ТИПА
}

\begin{abstract}
Аннотация. В работе для компьютерной программы контроля выполнения тестовых заданий расчетного типа открытой формы предложена теоретическая модель самообучения, основанная на использовании вероятности неверного выполнения заданий теста. Результаты имитационного моделирования и педагогических экспериментов, проведенных с использованием разработанной программы, реализующей алгоритм самообучения, показали обоснованность теоретической модели и целесообразность использования контролирующих самообучающихся программ для дифференцирования усилий студентов по выполнению заданий теста. Материалы статьи могут быть использованы для дальнейших исследований процесса самообучения компьютерных программ, реализующих тесты других типов и разработки тестов контроля знаний и умений на их базе.
\end{abstract}

Ключевые слова: контроль знаний; тестовое задание; компьютерная программа; имитационное моделирование; сложность заданий.

\section{1. ВВЕДЕНИЕ}

Динамика глобальных процессов в образовании, как и в целом в человеческом сообществе, характеризуется ускоренной интеллектуализацией всех их составляющих, использованием опыта предыдущих поколений, обобщенного посредством применения компьютерной техники. Одним из таких процессов является контроль знаний и умений при подготовке специалистов, объективность которого в современных условиях выдвигается на первый план, определяя в конечном итоге целесообразность и объем затрат общества на систему образования.

Постановка проблемы. «Для України проблема підготовки нової національної еліти набуває сьогодні особливої актуальності, оскільки всі наші негаразди значною мірою зумовлені саме вкрай недостатнім професіоналізмом фахівців» [1]. Основной составляющей профессионализма специалистов является объем усвоенных знаний и умение использовать их в практической деятельности. Оценка этой составляющей в национальной и международной практике образования проводится с применением тестов. Одним из важных аспектов применения тестов, отвечающий за их объективность, является учет сложности заданий тестов при оценивании их выполнения.

Анализ последних исследований и публикаций. Сопоставлению подготовленности респондентов и сложности заданий посвящено значительное количество научных и исследовательских работ. В [2; 3] такое сопоставление предлагается осуществлять с использованием критериев затраченного на выполнение заданий времени и количеству баллов, полученных респондентами при выполнении заданий теста. Значительных успехов в этом направлении достигнуто с использованием современной теории тестирования - Item Response Theory (IRT) [4]. В большинстве работ сложность заданий принимается как фиксированная величина (изначально 
определенная и/или скорректированная после выполнения теста в целом). На этом основании в дальнейшем судят о подготовленности респондентов и проводят оценивание их учебных достижений [4-6]. В [6] на основе анализа сложности заданий тестов разработан алгоритм корректировки баллов теста, необходимой для дифференциации участников тестирования, набравших одинаковые тестовые баллы. В работе [3] анализируется адаптивная модель тестирования, в которой тест приспосабливается под уровень знаний конкретного обучаемого по следующей схеме. «Если обучаемый правильно отвечает на тестовые задания, сложность последующих заданий повышается, если неправильно - понижается». Работа [5] посвящена оцениванию тестов в зависимости от уровня их сложности и включает технологию распределения тестовых заданий по уровням сложности, которая сводится к определению сложности тестовых заданий с использованием модифицированной современной теории тестирования IRT, исходя из экспериментальных данных предварительного тестирования. В монографии [2] рассматривается учет сложности в различных моделях тестов, оптимизация тестов по критерию сложности. В качестве меры сложности задания принято среднее число баллов, полученное всеми студентами, участвовавшими в тестировании, усредненное по всем вопросам. Относительно этой меры определяют сложность остальных вопросов. Учитывая, что среднее число баллов является случайной величиной, в работе вводятся доверительные границы, в которых возможно изменение среднего значения (базовой меры). Работа [7] посвящена дифференцированию усилий студентов в процессе усвоения языковых выражений применением специальной методики определения проектной сложности заданий и методики оценивания их выполнения.

Как видно из анализа источников, в настоящее время организация тестового контроля знаний определяется тенденцией разработки контролирующих программ, содержащих интеллектуальную составляющую: приспособлением к выявленным в процессе контроля данным. Цель этого процесса - создать равносложные для всех респондентов условия контроля с минимизацией затрат на его проведение и повышение объективности контроля учетом сложности заданий теста. Однако само значение сложности заданий в проводимых разработках принимается априори известным (или определяемым), что не соответствует реалиям процесса контроля.

Цель статьи. Разработка методики самообучения компьютерных программ контроля выполнения тестовых заданий расчетного типа открытой формы.

\section{2. МЕТОДЫ ИССЛЕДОВАНИЯ}

Исследования проводились в рамках оценивания перспективности разработки самообучающихся программ для контроля выполнения заданий расчетного типа. В процессе исследований использовались следующие методы: анализ теоретических источников по проблеме учета сложности заданий при оценивании результатов тестового контроля; имитационное моделирование; проведение педагогических экспериментов; анализ, обобщение и оценивание результатов.

\section{3. РЕЗУЛЬТАТЫ ИССЛЕДОВАНИЯ}

Как следует из проведенного анализа, значительные усилия теоретиковтекстологов и разработчиков программных продуктов контроля знаний направлены на учет сложности заданий, включаемых в тесты успешности. Это связано с тем, что учет фактора сложности является одним из основных моментов, обеспечивающих 
объективность процедуры контроля знаний. Понятие сложности заданий при выполнении различных тестов имеет свои особенности. В дальнейшем рассмотрении под сложностью задания будем понимать меру усилий респондента по верному выполнению этого задания. Усилия, прилагаемые разными респондентами для выполнения одного и того же задания сугубо индивидуальны, так как каждый респондент обладает индивидуальным набором психофизических факторов, влияющих на результат ответа и индивидуальным объемом усвоенных знаний, обеспечивающих достижение верного ответа. Отсюда следует, что данная мера имеет случайный характер и должна описываться в терминах теории вероятностей.

\section{1. Теоретическая модель самообучения}

Одной из характеристик случайной величины является вероятность ее появления [8]. Очевидно, что чем сложнее задание, тем больше вероятность его неверного выполнения, которую можем принять в качестве критерия сложности задания. Вероятность неверного выполнения заключена в интервале $0 \leq P_{n v} \leq 1$. Вариант $P_{n v}=0$ соответствует ситуации, при которой материал контрольного задания в полной мере изучен всеми респондентами и выявлен ими при контроле (все ответили верно). Вариант $P_{n v}=1$ соответствует ситуации, когда ответ на задание выходит за рамки выявленного каждым респондентом уровня знаний (никто не ответил верно). Оперировать непосредственно вероятностью неверного выполнения при оценивании результатов контроля неудобно. Поэтому при контроле для сопоставления сложности заданий теста между собой и некоторой шкалой оценивания результатов предлагается использовать коэффициент сложности заданий $S Z$ (аналог принятого в ряде работ набора баллов тестовых заданий), который может быть представлен в следующем виде:

$$
S Z_{i}=S Z_{\min }+\left(S Z_{\max }-S Z_{\min }\right) P_{n v, i}
$$

где $S Z_{i}$ - коэффициент сложности $i$-го задания теста; $S Z_{\min }-$ минимальное значение коэффициента сложности заданий в тесте; $S Z_{\max }-$ максимальное значение коэффициента сложности заданий в тесте; $P_{n v, i}$ - вероятность неверного выполнения $i$-го задания теста.

Минимальное и максимальное значение коэффициента сложности заданий теста устанавливаются при проектировании теста, в частности путем экспертной оценки [2]. При проектировании теста возможно использование относительных значений коэффициента сложности, например для $i$-го задания теста его относительное значение $O S Z_{i}$ равно:

$$
O S Z_{i}=\frac{S Z_{i}}{S Z_{\text {max }}}=\frac{S Z_{\text {min }}}{S Z_{\text {max }}}+\left(1-\frac{S Z_{\text {min }}}{S Z_{\text {max }}}\right) P_{n v, i}
$$

Если процесс тестирования организовать таким образом, что после каждого сеанса контроля, с использованием его результатов, производится расчет новых значений вероятности неверного выполнения $i$-го задания теста $P_{n v, i}$ (а значит, и сложности задания), то знания каждого следующего респондента будут оценены более объективно. Это означает, что контролирующая программа постоянно будет повышать свой «уровень экзаменатора», то есть самообучаться без участия ее разработчика (под самообучением понимается целенаправленное изменение под воздействием результатов контроля числовых характеристик программы, используемых при оценивании результатов контроля). 
Предложенная процедура определения сложности применима для тестовых заданий расчетного типа открытой формы, оценивание которых производится в формате «верно - неверно» с учетом сложности правильно выполненных заданий [9], так как значения вероятностей неверного выполнения могут быть достаточно просто определены в процессе контроля:

$$
P_{n v, i, j}=n_{i, j} / s_{i, j},
$$

где $P_{n v, i, j}$ - вероятность неверного выполнения $i$-го задания теста после проведения $j$-го сеанса контроля; $n_{i, j}$ - количество неверных выполнений $i$-го задания теста после проведения $j$-го сеанса контроля; $s_{i, j}$ - общее количество выполнений $i$-го задания теста после проведения $j$-го сеанса контроля.

Для проведения расчетов по (3) программе необходимо хранить информацию о количестве неверных выполнений и общем количестве выполнений каждого задания. Учитывая, что структура контролирующей программы [9] включает сохраняемый протокол контроля, постоянно изменяющаяся исходная информация для определения вероятности неверного выполнения задания теста может быть получена при минимальном объеме модификации кода базовой программы-оболочки [10].

\section{2. Имитационное моделирование процесса контроля}

Для выяснения особенностей предложенной процедуры рассмотрим теоретический вариант контроля, который заключается в том, что существует фиксированное, но заранее не известное распределение сложности заданий теста (истинная вероятность неверного выполнения заданий). В соответствии с этим распределением (в статистическом смысле) будут отвечать («верно - неверно») респонденты на задания теста. Тогда, независимо от проектного распределения сложности заданий, при корректировке сложности по результатам каждого выполнения (определения вероятности неверного выполнения задания) мы должны асимптотически приближаться к истинному распределению их сложности.

Так как выполнение задания определяется случайным значением $P_{n v, i, j}$, для определения результата тестирования мы можем применить компьютерное имитационное моделирование [11]. Моделирование проводилось в предположении, что задания ранжированы по возрастанию сложности (по возрастанию значений вероятности неверно выполнения) и истинное распределение вероятности неверного выполнения соответствует квадратичному закону вида $P_{n v}=0,000517 \cdot(n z-1)^{2}$, где $n z-$ номер задания (вид зависимости не имеет принципиального значения и выбран произвольно). Количество заданий было выбрано равным 45 (соответствует количеству заданий реального теста [9]).

Результаты компьютерного имитационного моделирования распределения вероятности неверного выполнения заданий теста показаны на рис. 1 (описание алгоритма программы имитационного моделирования приведено в подразделе 3.4).

Диаграммы, показанные на рисунке, подтверждают правильность приведенных теоретических рассуждений. В качестве проектного распределения вероятности неверного выполнения (рис. 1а) принималось равномерное распределение, независимое от истинного распределения. С увеличением количества итераций $k i$ (для реальной ситуации - сеансов контроля) отклонение распределения вероятностей от их истинного распределения уменьшается (рис. 1б, 1в, 1г), что позволяет считать предложенную процедуру корректировки сложности заданий сходящимся процессом. Это означает, что в конечном итоге компьютерная программа «научится» оценивать 
выявленные знания респондентов в соответствии с истинным распределением сложности заданий.

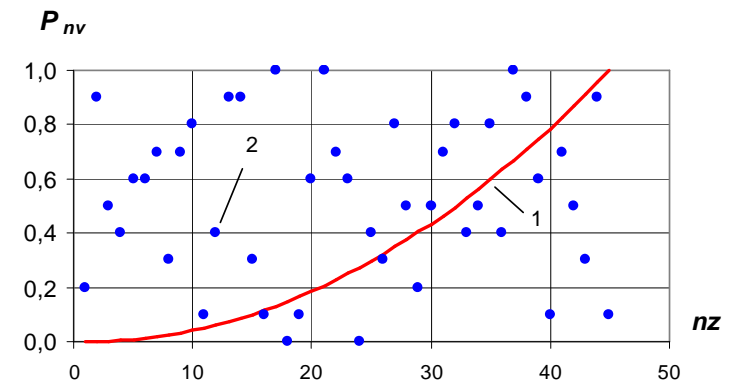

а) проектное распределение

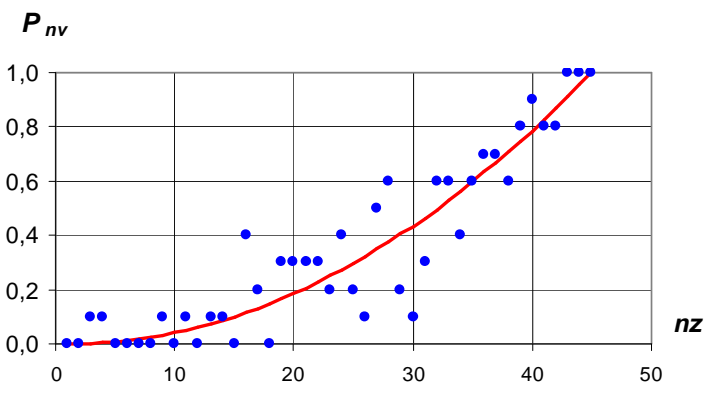

в) распределение при $k i=10$

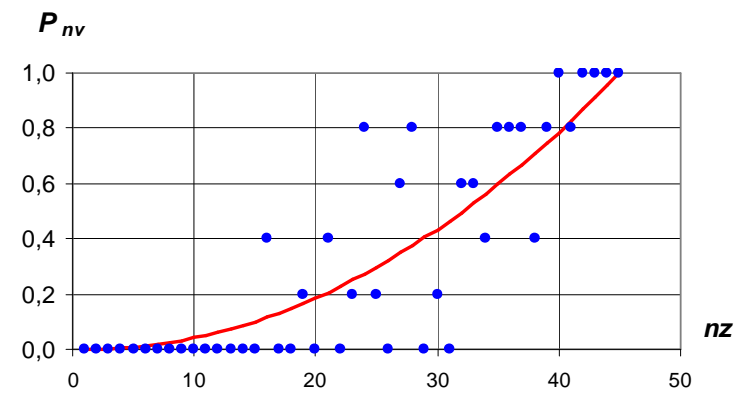

б) распределение при $\mathrm{ki}=5$

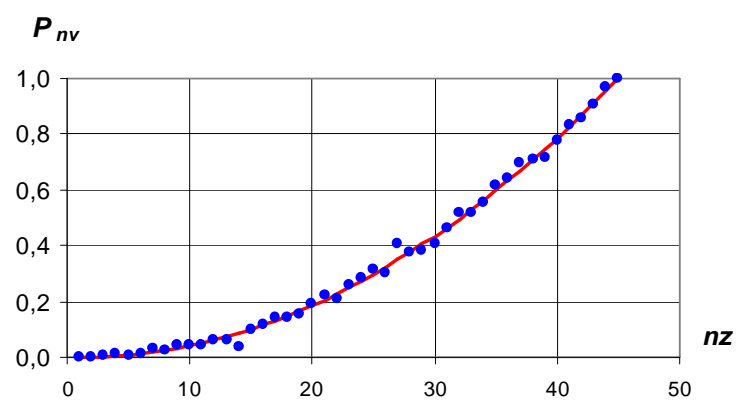

2) распределение при $k i=200$

Рис. 1. Изменение распределения вероятностей неверного выполнения 1 - истинное распределение; 2 - результаты имитаџионного моделирования

\section{3. Результаты педагогических экспериментов}

В реальной ситуации проверки знаний, в отличие от рассмотренного теоретического варианта, истинное распределение вероятностей не только неизвестно, но и не фиксировано, так как представляет собой случайную функцию, которая генерируется респондентами в процессе контроля.

Проведенный анализ результатов 55 выполнений тестовых заданий позволил получить следующие данные по распределению вероятности неверного выполнения, показанные на рис. 2 (зависимость вероятности $P_{n v}$ от среднего количества выполнения заданий в тесте $N$ ).

Из рисунка видно, что для заданий с малой вероятностью неверного ответа (малой сложностью) стабилизация значений вероятности происходит достаточно монотонно (рис. 2a), без значительных скачков, а для сложных заданий имеют место процессы переколебаний при приближении к стационарным значениям вероятности (рис. 2б). Быстрая стабилизация значений для малой вероятности неверного ответа объясняется тем, что респонденты делают меньше ошибок при ответах на более простые задания. Наличие значительных скачков вероятности при выполнении сложных заданий должно быть учтено, так как может значительно повлиять на объективность оценивания выполнения теста в целом. Сглаживание резких флуктуаций данных целесообразно проводить использованием средних значений вероятности (рис. 2в).

Таким образом, данные имитационного моделирования и результаты педагогического эксперимента свидетельствуют о том, что предложенная процедура корректировки вероятности неверного выполнения задания (корректировки сложности 
задания) является сходящимся процессом и может быть использована для самообучения контролирующей программы.

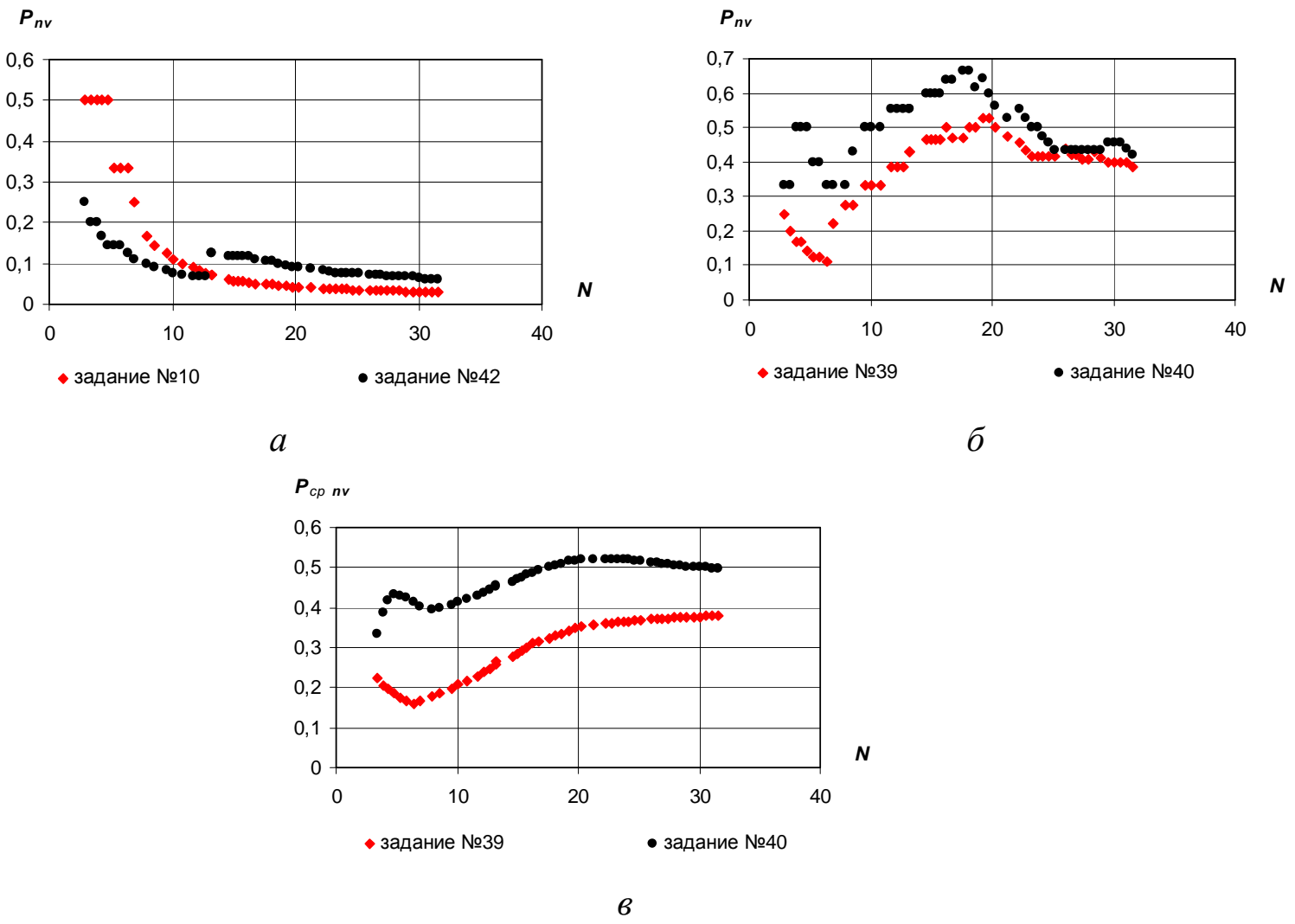

Рис. 2. Изменение вероятности неверного выполнения заданий по данным педагогического эксперимента

Оценка влияния на результаты тестового контроля респондентов процесса самообучения программы, проведенная с использованием реальных результатов тестирования [9] показали, что корректировка сложности заданий в процессе самообучения программы приводит к увеличению коэффициента выполнения для 58 \% респондентов, к уменьшению коэффициента выполнения для $36 \%$ респондентов, в остальных случаях результат практически не изменился.

\section{4. Описание алгоритма программы моделирования контроля}

Для имитационного моделирования процесса контроля знаний используем основные принципы, изложенные в [11; 12]. Тогда, применительно к структуре программы [9] имитационное моделирование, результаты которого приведены в п. 3.2, производится в следующем порядке. Для каждой $j$-ой итерации $(j=1,2,3, \ldots)$, которая моделирует ответ респондента и обработку результатов контроля:

- исходя из распределения вероятности неверного выполнения заданий, для каждого из них определяется факт его выполнения в формате «верно неверно»;

- для каждого $i$-го задания теста определяется общее количество выполнений $s_{i, j}$ и количество неверных выполнений $n_{i, j}$;

- по соотношению (3) вычисляется вероятность неверного выполнения $i$-го задания теста $P_{n v, i, j}$;

- по соотношению (1) или (2) корректируется коэффициент сложности заданий; 
- строится график, показанный на рис. 1.

Данная процедура повторяется до выявления основных тенденций процесса контроля.

Особенностью процедуры является пункт определения факта выполнения заданий в формате «верно - неверно». Как было показано выше, вероятность неверного выполнения изменяется в интервале от нуля до единицы (рис. 3). Тогда, используя генератор случайных чисел $X$ равномерно распределенных в интервале $[0,1)$, для определения факта «выполнение - невыполнение» задания можем применить следующую процедуру:

- задание выполнено неверно, если случайное число принадлежит интервалу $X \in\left[0, P_{n v, i}\right] ;$ на рис. 3 данный вариант представляет число $a$;

- задание выполнено верно, если случайное число принадлежит интервалу $X \in\left(P_{n v, i}, 1\right) ;$ на рис. 3 данный вариант представляет число $b$.

Шкала вероятности неверного выполнения задания

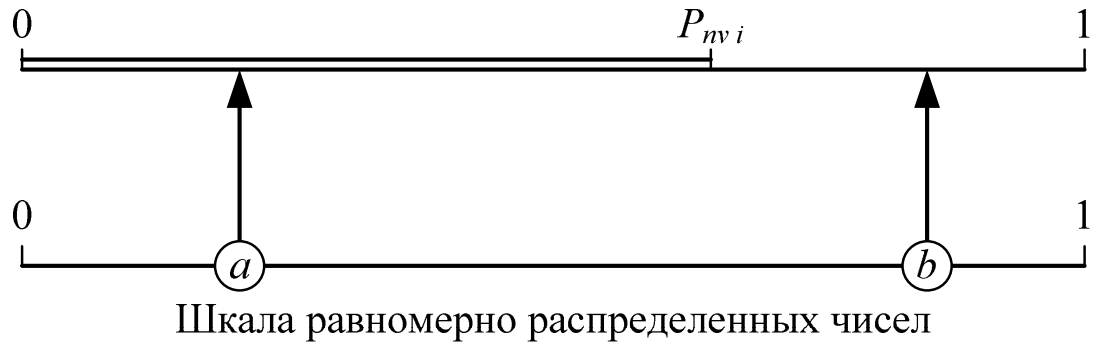

Рис. 3. Моделирование результата выполнения тестового задания

Данная процедура не учитывает изменение вероятности неверного выполнения заданий $P_{n v, i}$ в процессе контроля за счет изменения психофизического состояния респондентов, а также за счет эффекта «научаемости» (результат контроля зависит от того, в какой последовательности будут предъявляться задания). В виду случайного выбора заданий эти факторы не повлияют на основную тенденцию процесса контроля, а лишь замедлят процесс сходимости в теоретической модели контроля.

Практически в соответствии с описанным алгоритмом была проведена обработка педагогических экспериментов, результаты которой приведены в п. 3.3. Отличие состояло в том, что генератором факта выполнения заданий в формате «верно неверно» являлись данные реального контроля знаний, которые автоматически учитывают влияние факторов, рассмотренных выше.

\section{4. ВЫВОДЫ И ПЕРСПЕКТИВЫ ДАЛЬНЕЙШИХ ИССЛЕДОВАНИЙ}

1. Предложена теоретическая модель самообучения компьютерной программы контроля выполнения тестовых заданий расчетного типа открытой формы, основанная на использовании вероятности неверного выполнения заданий теста.

2. Результаты имитационного моделирования и педагогических экспериментов, проведенных с использованием разработанной программы, реализующей алгоритм самообучения, показали обоснованность теоретической модели и целесообразность использования контролирующих самообучающихся программ для дифференцирования усилий студентов по выполнению заданий теста. 
Материалы статьи могут быть использованы для дальнейших исследований процесса самообучения компьютерных программ, реализующих тесты других типов и разработки тестов контроля знаний и умений на их базе.

\section{СПИСОК ЛИТЕРАТУРЫ}

1. Романовський О. Г. Формування гуманітарно-технічної еліти в освітньому просторі Національного технічного університету "Харківський політехнічний інститут" / О. Г. Романовський, Ю. І. Панфілов // Сучасні педагогічні технології в освіті : збір. наук.-метод. праць / ред. О. Г. Романовський, Ю. І. Панфілов. - Харків : НТУ «ХПІ», 2012. - С. 13-23.

2. Алексеев А. Н. Дистанционное обучение инженерным специальностям : монография / А. Н. Алексеев. - Сумы : ИТД «Университетская книга», 2005. - 333 с. - ISBN 966-680-235-X.

3. Дуплик С. В. Модели педагогического тестирования [Електронный ресурс] / С. В. Дуплик // Казанский Государственный Технический университет им. А. Н. Туполева. - Режим доступа : http://www.dupliksv.hut.ru/pauk/papers/testmodel.html.

4. Ким В. С. Тестирование учебных достижений : монография / В. С. Ким. - Уссурийск : Издательство УГПИ, 2007. — 214 с. — ISBN 978-5-86733-165-8.

5. Технология оценивания тестов в зависимости от типа и уровня сложности тестовых заданий на основе интегрированной модели [Електронный ресурс] / [М. Бондаренко, В. Семенец, Н. Белоус, и др.]. - Режим доступа : http://www.uran.donetsk.ua/ masters/2013/fknt/sedakov/library/ibs-12p07.pdf.

6. Раков С. А. Алгоритм корректировки тестовых баллов на основе анализа сложности заданий / С. А. Раков, М. С. Мазорчук, Е. О. Бондаренко // Інформаційні технології в освіті. - 2013. № 16. - C. 49-56.

7. Петков А. А. Определение сложности заданий при контроле усвоения языковых выражений [Электронный ресурс] / А. А. Петков, М. А. Петкова // Інформаційні технології і засоби навчання. - 2014. - Том 40. - №2. - С. 126-134. - Режим доступу : http://journal.iitta.gov.ua/index.php /itlt/article/view/876/649.

8. Вентцель Е. С. Теория вероятностей / Е. С. Вентцель. — М. : Высш. шк., 2002. - 575 с.

9. Петков А. А. Тестовый контроль выполнения расчетных заданий на алгоритмическом уровне учебной деятельности [Электронный ресурс] / А. А Петков, М. А. Петкова // Інформаційні технології і засоби навчання. - 2014. - Том 43. - №5. - С. 189-200. - Режим доступа : http://journal.iitta.gov.ua/index.php/itlt/article/view/1121/837.

10. Петков А. А. "Компьютерная программа оценки уровня владения умениями применять теоретические знания для расчетов в конкретной предметной области "Расчет". Свидетельство о регистрации авторского права на произведение № 37534 от 23.03.2011. Государственный департамент интеллектуальной собственности Министерства образования и науки Украины.

11. Майер Р. В. Кибернетическая педагогика : Имитационное моделирование процесса обучения / Р. В. Майер. - Глазов : ГГПИ, 2013. - 138 с.

12. Майер Р. В. Компьютерное моделирование : учебник для студентов педагогических вузов / Р. В. Майер. - Глазов : ГГПИ, 2014. - 531 с.

Материал поступил в редакцию 26.01.2015 2.

\section{САМОНАВЧАНІСТЬ КОМП'ЮТЕРНИХ ПРОГРАМ КОНТРОЛЮ ВИКОНАННЯ ТЕСТОВИХ ЗАВДАНЬ РОЗРАХУНКОВОГО ТИПУ}

\section{Пєтков Олександр Олександрович}

старший науковий співробітник, доктор технічних наук, професор кафедри «нженерна електрофізика» Національний технічний університет «Харківський політехнічний інститут», м. Харків, Україна apetkov@yandex.ru

Анотація. У роботі для комп'ютерної програми контролю виконання тестових завдань розрахункового типу відкритої форми запропонована теоретична модель самонавчання, заснована на використанні ймовірності невірного виконання завдань тесту. Результати імітаційного моделювання й педагогічних експериментів, проведених 3 використанням 
розробленої програми, що реалізує алгоритм самонавчання, показали обгрунтованість теоретичної моделі й доцільність використання контролюючих програм, що самонавчаються, для диференціювання зусиль студентів по виконанню завдань тесту. Матеріали статті можуть бути використані для подальших досліджень процесу самонавчання комп'ютерних програм, що реалізують тести інших типів і розробки тестів контролю знань і вмінь на їхній базі.

Ключові слова: контроль знань; тестове завдання; комп'ютерна програма; імітаційне моделювання; складність завдань.

\title{
SELF-LEARNING OF COMPUTER PROGRAMS FOR CONTROLLING TEST TASKS EXECUTION OF CALCULATED TYPE
}

\author{
Oleksandr O. Petkov \\ senior researcher, Doctor of technical sciences, professor of the Department "Engineering Electrophysics" \\ National Technical University "Kharkov Polytechnic Institute", Kharkov, Ukraine \\ apetkov@yandex.ru
}

\begin{abstract}
In this paper the theoretical model of self-learning based on the use of probability of incorrect assignments of the test for a computer program for monitoring the implementation of tests as the open form is suggested. The results of simulation modeling and pedagogical experiments conducted by using the developed program which implement the self-learning algorithm, are showed the validity of the theoretical model and the appropriateness using of selflearning control programs for differentiation efforts of students on the realization of these tests. The article's submissions may be used for further studies of the self-training process of software that realize other types of tests and test development of knowledge control and ability based on them.
\end{abstract}

Keywords: control of knowledge; test task; computer software; simulation modeling; the complexity of the tasks.

\section{REFERENCES (TRANSLATED AND TRANSLITERATED)}

1. Romanovskiy O. G. Formation of humanitarian-technical elite in educational environment of the National Technical University "Kharkiv Polytechnic Institute" / O. G. Romanovskiy, Yu. I. Panfllov // Suchasni pedagogIchni tehnologiyi v osviti: zbir. nauk.-metod. prats / red. O. G. Romanovskiy, Yu. I. Panfilov. — Harkiv: NTU «HPI», 2012. (in Ukrainian)

2. Alekseev A. N. Remote training to engineering specialities : monografija / A. N. Alekseev. Sumy : ITD «Universitetskaja kniga», 2005. — 333 s. — ISBN 966-680-235-X. (in Russian)

3. Duplik S. V. Models of pedagogical testing [online] / S. V. Duplik // Kazanskij Gosudarstvennyj Tehnicheskij universitet im. A.N. Tupoleva - Available from : http://www.dupliksv.hut.ru/pauk/papers/testmodel.html. (in Russian)

4. Kim V. S. Testing of educational achievements : monografija / V. S. Kim. — Ussurijsk : Izdatel'stvo UGPI, 2007. — 214 s. — ISBN 978-5-86733-165-8. (in Russian)

5. Technology of estimation tests depending on type and a level of complexity of test tasks on the basis of the integrated model [online] / [M. Bondarenko, V. Semenec, N. Belous, V. Borisenko, I. Kucevich, I. Belous, O. Melezhik]. - Available from : http://www.uran.donetsk.ua/ masters/2013/fknt/sedakov/library/ibs-12-p07.pdf. (in Russian)

6. Rakov S. A. Algorithm of updating of test points on the basis of the analysis of complexity of tasks / S. A. Rakov, M. S. Mazorchuk, E. O. Bondarenko // Informacijni tehnologiï v osviti. 2013. — № 16. - S. 49-56. (in Russian)

7. Petkov A. A Definition of complexity of tasks at the control of mastering of linguistic expressions [online] / A. A. Petkov, M. A. Petkova // Informacijni tehnologiï i zasobi navchannja. — 2014. Tom 40. - № 2. - ․ $\quad$ S. 126-134. - Available from : http://journal.iitta.gov.ua/index.php/itlt/article/view/876/649. (in Russian)

8. Ventcel E. S. Probability theory / E. S. Ventcel. — M. : Vyssh. shk., 2002. — 575 p. (in Russian)

9. Petkov A. A Test control implementation calculated jobs algorithmic level of educational activity [online] / A. A. Petkov, M. A. Petkova // Informacijni tehnologiï i zasobi navchannja. — 2014. — 
Tom 43. - № 5. - ․ S. 189-200. - $\quad$ S. Available from : http://journal.iitta.gov.ua/index.php/itlt/article/view/1121/837. (in Russian)

10. Petkov A. A. "Computer program evaluation proficiency skills to apply theoretical knowledge to the calculations in a particular domain" Calculate ". Certificate of registration of copyright in a work \# 37534 dated 23.03.2011.Gosudarstvennyy departament intellektualnoy sobstvennosti Ministerstva obrazovaniya i nauki Ukrainy (in Russian)

11. Mayer R. V. Cybernetic Pedagogy: Simulation modeling of the learning process / R. V. Mayer. Glazov: GGPI, 2013. - 138 s. (in Russian)

12. Mayer R. V. Computer modeling: A textbook for students of pedagogical universities / R. V. Mayer. - Glazov: GGPI, 2014. - 531 s. (in Russian) 